ELECTRONIC RESEARCH ANNOUNCEMENTS OF THE AMERICAN MATHEMATICAL SOCIETY

Volume 3, Pages 78-82 (August 21, 1997)

S 1079-6762(97)00026-7

\title{
PROOF OF CONWAY'S LOST COSMOLOGICAL THEOREM
}

\author{
SHALOSH B. EKHAD AND DORON ZEILBERGER
}

(Communicated by Ronald Graham)

\begin{abstract}
John Horton Conway's Cosmological Theorem about sequences like $\mathbf{1}, \mathbf{1 1}, \mathbf{2 1}, \mathbf{1 2 1 1}, \mathbf{1 1 1 2 2 1}, \mathbf{3 1 2 2 1 1}, \ldots$, for which no extant proof existed, is given a new proof, this time hopefully for good.
\end{abstract}

One of the most intriguing sequences $[\mathrm{CG}],[\mathrm{F}],[\mathrm{SP}],[\mathrm{V}]$ is Conway's $[\mathrm{C}] \mathbf{1}, \mathbf{1 1}$, $21,1211,111221,312211,13112221,1113213211, \ldots$. It is defined by the rule $C_{0}:=1$, and $C_{i}:=J H C\left(C_{i-1}\right)$, for $i>0$, where $J H C$ is Conway's audioactive operator:

$$
J H C\left(a_{1}^{m_{1}} a_{2}^{m_{2}} \ldots a_{r}^{m_{r}}\right):=m_{1} a_{1} m_{2} a_{2} \ldots m_{r} a_{r} .
$$

Here $a^{m}$ is shorthand for " $a$ repeated $m$ times" (and we agree that the description is optimal, i.e. $a_{i} \neq a_{i+1}$ ). We assume familiarity with Conway's charming article $[\mathrm{C}]$.

Conway proved that his sequence has the property length $\left(C_{i+1}\right) /$ length $\left(C_{i}\right) \rightarrow$ $\lambda$, where $\lambda=1.303577269 \ldots$ is Conway's constant. He also stated that, more generally, if one starts with an arbitrary nonempty finite string of integers, $B_{0}$ (except 'boring old 22'), and defines $B_{i}:=J H C\left(B_{i-1}\right), i>0$, then still

$$
\text { length }\left(B_{i+1}\right) / \text { length }\left(B_{i}\right) \rightarrow \lambda \text {. }
$$

This is an immediate consequence of

The Cosmological Theorem. There exists an integer $N$ such that every string decays in at most $N$ days to a compound of common and transuranic elements.

Conway stated that two independent proofs used to exist, one by himself and Richard Parker (that only proved that $N$ existed), and another one by Mike Guy (that actually proved that one may take $N=24$, and that it was best possible). Unfortunately both proofs were lost. Here we announce a new proof (which establishes that one may take $N=29$; with more computations one should be able to rederive (or else refute) Guy's sharp $N=24$ ).

The Cosmological Theorem is an immediate consequence of the following lemma.

Lemma. The length of any atom in the splitting of a 9-day-old string is $\leq 80$. Every such atom decays, in at most 20 days, into stable or transuranic elements.

Received by the editors May 6, 1997.

1991 Mathematics Subject Classification. Primary 05Axx.

Supported in part by the NSF. 
The lemma is proved by typing Cosmo (8) ; in the Maple package HORTON, retrievable from http://www . ams .org/era/1997-03-11/S1079-6762-97-00026-7/html/HORTON.

The procedure Cosmo computes iteratively all non-splittable strings of length $i$ $(i=1,2, \ldots)$ that might conceivably be substrings ('chunks') of an atom in the splitting of a 9-day-old-string (by backtracking, examining its possible ancestors up to (at most) 8 days back and rejecting those that lead to grammatically incorrect ancestors; see examples below). Every time a string of length $i$ is accepted, its longevity (number of days it takes to decay to stable or transuranic elements) is computed, and checked whether it is finite. The maximal longevity turned out to be 20. The program halts if and when an $i$ is reached for which the set of such conceivable strings of length $i$ is empty.

If the program halts (it did for us), then the Lemma, and hence the Cosmological Theorem, are proved. In fact it halted after $i=80$, implying that there do not exist atoms of length $>80$ that occur in the splitting of mature (i.e. 9-day-old) strings, and that all the atoms have bounded $(\leq 20$, as it turned out) longevity. We also get that the longevity of an arbitrary string is $\leq 9+20=29$. The input and output files may be obtained from

http://www . ams.org/era/1997-03-11/S1079-6762-97-00026-7/html/incosmo and

http://www . ams .org/era/1997-03-11/S1079-6762-97-00026-7/html/ocosmo respectively.

The Maple package HORTON, also available from the authors' websites (http://www.math.temple.edu/ zeilberg and http://www.math.temple.edu/ ${ }^{\sim}$ ekhad) rederives many other results in Conway's paper, in particular it finds all the stable elements $a b$ initio, finds the minimal polynomial for $\lambda$, finds the abundance of all the stable elements, and computes the longevity of any string. We refer the reader to the on-line documentation and to the source code.

\section{Details}

Recall that Conway proved that it suffices to consider strings on $\{1,2,3\}$. Let's call a chunk that starts with a comma female, and a chunk that does not, male. Any chunk could have come either from a father or a mother (but of course not from both). Define

$$
\text { ParentOfGirl }\left(a_{1} a_{2} a_{3} a_{4} a_{5} a_{6} \ldots\right):=a_{2}^{a_{1}} a_{4}^{a_{3}} a_{6}^{a_{5}} \ldots,
$$

and

$$
\text { ParentOfBoy }\left(a_{1} a_{2} a_{3} a_{4} a_{5} a_{6} \ldots\right):=a_{1} a_{3}^{a_{2}} a_{5}^{a_{4}} \ldots .
$$

Since a parent of a chunk may be either female or male (but we have no way of knowing), any chunk has two potential parents (but of course only one actual one), (up to) four (potential) grandparents (some of them may disqualify on the grounds of being grammatically incorrect), and so on. Now there are lots of chunks that cannot possibly be factors of a mature string. Take for example the female chunk ", 12, 32,". It cannot be a chunk of a 1-day-old string (as observed in [C]), since starting at day 1 , all strings are descriptive, and ", 12, 32," would have been 
abbreviated ", 42 ,". So we can eliminate from the outset any female string of the form ", $a c, b c$,", in strings that are older than 0 days.

Now consider the female string ", 32,33 ,". It is grammatically correct, and so can be a chunk of a 1-day-old string. Her parent is: "222333". If the parent is a father, then it is punctuated " $2,22,33,3$ ", which is grammatically incorrect, and if the parent is a mother, then it would be ", $22,23,33$,", that is equally grammatically incorrect. Hence we can conclude that ", 32,33 ,", while it may be a chunk of an atom in the splitting of a 1-day-old string, cannot possibly be such a chunk of a 2-day-old string.

Consider on the other hand the female string ", 12,21, ,. Her father is " 2,11 ", and her mother is ", 21,". Her paternal grandparent is 21 who is OK, being twolettered. Hence we cannot rule out ", 12,21 ," as a possible chunk in an $L$-day-old string (for any $L>0$ ).

Let us define $U_{L}(i)$ as the set of female strings on the alphabet $\{1,2,3\}$ of length $2 i$ that do not split, and that have at least one $(\text { great })^{L-2}$ grandparent

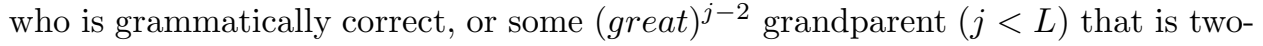
lettered. In order to find $U_{L}(i)$, we take all the survivors that made it to $U_{L}(i-1)$, append all the nine possible endings $11,12,13,21,22,23,31,32,33$, and see which of the resulting female strings do not split and in addition survive up-to-depth$L$ genealogical screening. Whenever we induct a new member to $U_{L}(i)$, we also compute her longevity, and the longevity of her male extensions of length $2 i+1$ : " $1, w$ ", " $2, w$ ", " $3, w$ ", her female extensions of length $2 i+1:$ " $w, 1$ ", " $w, 2$ ", " $w, 3$ ", and her nine male extensions of length $2 i+2$ : " $1, w, 1$ ", " $1, w, 2$ ", " $1, w, 3$ ", " $2, w, 1$ ", " $2, w, 2$ ", " $2, w, 3$ ", " $3, w, 1$ ", " $3, w, 2$ ", " $3, w, 3$ ”. We always keep track of the maximum longevity to-date.

If for some $L$ ( $L=8$ worked), and some $i$ (we got $i=40$ ), $U_{L}(i)=\emptyset$, and the longevity record, $M$, is finite (we got $M=20$ ), then it follows that any string $w$ decays into stable or transuranic elements in at most $M+L+1$ days. Indeed, $w^{\prime}:=J H C^{9}(w)$ is a 9-day-old string. Split $w^{\prime}$ into atoms. We know that each of these atoms has length $\leq 80$. Indeed, if such an atom were a female of even length $\geq 82$, then her head, consisting of the first 80 letters, would have been a member of $U_{8}(40)$, contradicting the fact that this set is empty. Similarly if such an atom were a female of odd length, or a male, then an appropriate factor would be a member of the empty $U_{8}(40)$. So every atom in the splitting of $w^{\prime}$ has length $\leq 80$. Furthermore, all these atoms either belong to $U_{L}(i)$ for some $i<40$, or have one of the forms $1 v, 2 v, 3 v, v 1, v 2, v 3,1 v 1,1 v 2,1 v 3,2 v 1,2 v 2,2 v 3,3 v 1,3 v 2,3 v 3$, where $v \in U_{L}(i)$, for some $i \leq 39$. But all these strings were tested for finite longevity by Cosmo, and turned out to have longevity $\leq 20$. Since each of the atoms in the splitting of $w^{\prime}$ decays in at most 20 days, so does $w^{\prime}$, and hence $w$ decays in at most $20+8+1=29$ days.

\section{ON A POSTERIORI TRIVIAL THEOREMS: THE Ultimate PROOF OF THE Four-Color TheOREM SHOULD EMUlate our PROOF}

Some statements are a priori trivial (regardless of whether they are true or false), for example that there do not exist projective planes of order 10 (proved by Clement Lam), or the (still open) conjecture that White can always win at Chess. Of course by trivial we mean modulo a finite amount of calculations. 
Other statements are only a posteriori trivial. Many conjectures seem only to be a posteriori trivial if they turn out to be false, and are rendered trivial by exhibiting a counterexample; for instance, Euler's conjecture that $A^{4}+B^{4}+C^{4}=D^{4}$ is unsolvable, disproved by Elkies. Of course, in the Gödelian sense, every decidable statement is a posteriori trivial. Both proof and disproof, being finite, could be eventually found by exhaustive search.

However, the Cosmological Theorem turned out to be a posteriori trivial in a more genuine 'object-oriented' sense. We wrote a program that iteratively computes $U_{L}(i)$, and a proof would be obtained if $U_{L}(i)$ is empty for some $L$ and $i$. A priori, we have no way of knowing whether we would be successful. If our civilization would die, or more realistically, the program would run out of memory, we would never know whether it was 'never' or 'not yet'. All we can do is hope. Also, suppose that the program does not halt. In that case it does not mean that the statement of the conjecture is false. All it means is that our particular approach failed. So you only win if and when the proof-program halts. But, once that happens, in order to check the validity of our proof, it is a waste of time to examine the members of $U_{8}(i)$ for $i=1,2,3, \ldots$, and to examine the decay process for each accepted chunk. All we need is to check the computer program. Once the logic of the program has been checked, all we have to do is, bet on an $L$, say $L=8$, and type Cosmo (8); , and hope that it would halt in our lifetime. If it does, that is all there is to it, and we have a one-line proof.

A celebrated example of an a posteriori trivial theorem is Appel and Haken's Four-Color-Theorem. Their approach was to find a finite unavoidable set of reducible configurations. The original proof [AHK] used an excessive amount of human effort. This was considerably improved in the new proof by Robertson, Sanders, Seymour, and Thomas [RSST], but this is not the ultimate proof. Eventually one should be able to type Prove4CT(); , and the truth of the theorem should be implied by the halting of the program. In order to check the validity, the checker would not need to see any specific configuration. Everything should be done internally and silently by the computer. All that the checker would have to do is check the program.

And who knows? Perhaps the nonexistence of odd perfect numbers, the $3 n+1$ problem, the Riemann Hypothesis, etc. etc. are all a posteriori trivial? Let us hope that their proof-programs will halt in our lifetime.

\section{ACKNOWLEDGEMENT}

We wish to thank John Conway, Steve Finch, and Ilan Vardi for helpful remarks on an earlier version.

\section{REFERENCES}

[AHK] K. Appel, W. Haken, and J. Koch, Every planar map is four-colorable, Illinois J. Math. 21 (1977), 429-567. MR 58:27598a,b

[C] J. H. Conway, The weird and wonderful chemistry of audioactive decay, Open Problems in Communication and Computation (T.M. Cover and B. Gopinath, eds.), Springer, 1987, pp. 173-188. MR 89c:94003

[CG] J. H. Conway and R. K. Guy, The book of numbers, Copernicus, New York, 1996. CMP 97:02

[F] S. Finch, Favorite Mathematical Constants Website, http://www. mathsoft. com/asolve/constant/cnwy/cnwy.html. 
[RSST] N. Robertson, D. P. Sanders, P. Seymour, and R. Thomas, A new proof of The Four-Color Theorem, ERA Amer. Math. Soc. 2 (1996), 17-25.

[SP] N. J. A. Sloane and S. Plouffe, The encyclopedia of integer sequences, Academic Press, San Diego, CA, 1995. MR 96a:11001

[V] I. Vardi, Computational recreations in Mathematica, Addison-Wesley, 1991. MR 93e:00002

Department of Mathematics, Temple University, Philadelphia, PA 19122

E-mail address: ekhad@math.temple.edu

Department of Mathematics, Temple University, Philadelphia, PA 19122

E-mail address: zeilberg@math.temple.edu 